\title{
Uso de equipamentos de monitoramento da saúde por idosos no ambiente doméstico
}

\author{
The use of health-monitoring devices by elderly in the household
}

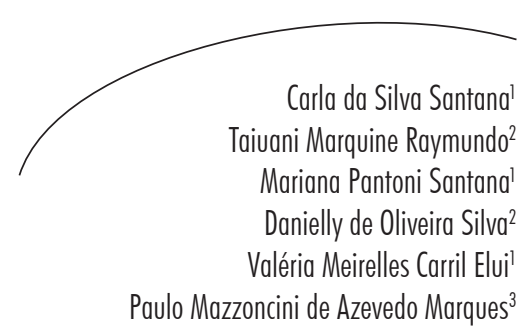

Resumo

Introdução: $\mathrm{O}$ envelhecimento da população aumenta a demanda por cuidados de saúde e o progresso nesta área tem sido ampliado com as novas tecnologias de cuidado. A população brasileira tem incorporado equipamentos de monitoramento à saúde de uso doméstico e estes podem auxiliar o sujeito se este for adequadamente instrumentalizado para o uso. Objetivo: Identificar os aparelhos de monitoramento da saúde que são frequentemente utilizados por idosos em ambiente doméstico, descrevendo as dificuldades apresentadas na utilização destes equipamentos. Método: Trata-se de estudo exploratório, analítico e transversal. A coleta de dados incluiu um questionário socioeconômico, Escala de Lawton \& Brody para avaliação da capacidade funcional e um questionário para classificação do uso de equipamentos eletrônicos por idosos. Para a análise dos dados, foi utilizado o método de estatística descritiva. Resultados: Participaram do estudo 185 idosos ativos, sendo 78,4\% mulheres. Do total dos participantes, 53\% eram casados e 31,9\% possuíam ensino superior completo. Quanto aos problemas de saúde e uso de dispositivos, 48,5\% relataram ter hipertensão arterial sistêmica e $25,3 \%$, diabetes; $38,4 \%$ relataram possuir o aferidor de pressão arterial digital, $14 \%$ o medidor de glicemia e $15,7 \%$ o porta-comprimidos. Entre as dificuldades, estavam o manuseio do aferidor de pressão e do medidor de glicemia, a falta de conhecimento na interpretação e gravação dos resultados no aparelho e, por não confiarem nos resultados, os idosos referiram preferir recorrer ao auxílio de um profissional qualificado. Conclusão: As dificuldades de uso apontadas podem estar associadas ao fato de os idosos de hoje pertencerem a uma geração que não teve contato com estes dispositivos quando mais jovens, pela falta de treinamento para uso e pela falta de conhecimentos para gerenciar os resultados.

Departamento de Neurociências e Ciências do Comportamento, Faculdade de Medicina de Ribeirão Preto. Universidade de São Paulo. Ribeirão Preto, SP, Brasil.

2 Programa de Pós-graduação Interunidades em Bioengenharia. Universidade de São Paulo. São Carlos, SP, Brasil.

3 Departamento de Clínica Médica, Faculdade de Medicina de Ribeirão Preto. Universidade de São Paulo. Ribeirão Preto, SP, Brasil.

Correspondência / Correspondence

Carla da Silva Santana

E-mail: carla.santana@fmrp.usp.br

Palavras-chave: Doença Crônica. Saúde do Idoso. Desenvolvimento Tecnológico. Autocuidado. 


\section{Abstract}

Introduction: The aging population increases the demand for health care and progress in this area has been driven by new care-related technologies. The Brazilian population has incorporated the use of health-monitoring equipment in the domestic environment and these can assist subjects if they receive adequate training. Objective: Identify health-monitoring devices that are often used by the elderly in the home environment and describe the difficulties encountered in the use of these devices. Method: This is an exploratory, analytical and cross-sectional study. Data collection included a socioeconomic questionnaire, a Lawton and Brody Scale to assess functional capacity, and a questionnaire to classify the use of electronic devices by elderly. Data analysis employed the method of descriptive statistics. Results: The study included 185 active seniors, of which $78.4 \%$ were women. Of the total participants, $53 \%$ were married and $31.9 \%$ have completed higher education. As for health problems and use of devices, $48.5 \%$ reported having hypertension and $25.3 \%$ diabetes; $38.4 \%$ reported owning a digital blood pressure meter; 14\%, a glucose meter and 15.7\%, pillboxes. Among the difficulties listed were handling the digital blood pressure meter and blood glucose meter and lack of knowledge in the interpretation and recording of results. The subjects also reported not trusting the results of the devices and preferring to seek help from a qualified professional. Conclusion: The difficulties of use may be associated with the fact that today's seniors belong to a generation that had no contact with these devices when younger, lack of training to use the equipment and lack of knowledge of how to manage results.
Key words: Chronic Disease. Health of the Elderly, Technological Development. Self Care.

\section{INTRODUÇÃO}

O processo de envelhecimento altera o equilíbrio entre saúde e doença. Há um aumento na fragilidade de alguns idosos, assim como na vulnerabilidade física e funcional. Os idosos frágeis apresentam-se susceptíveis a terem doenças crônicas que limitam suas atividades cotidianas, apresentam alterações biológicas e diminuição da capacidade imunológica, assim como limitações vasculares, neurológicas e sensoriais.

As doenças crônicas não transmissíveis (DCNT) são consideradas hoje um problema global, além de uma ameaça à saúde e ao desenvolvimento humano. ${ }^{2}$ De acordo com a Organização Mundial da Saúde (OMS), em 2008 um estudo que incluiu informações de 193 países mostrou que as DCNTs foram responsáveis por 36 milhões de óbitos, sendo consideradas a maior causa de morte em todo o mundo. De acordo com o estudo, as doenças cardiovasculares foram responsáveis por $48 \%$ das mortes no grupo, seguidas por câncer $(21 \%)$, doenças respiratórias crônicas $(12 \%)$ e diabetes $(3 \%){ }^{3}$

Considerando que as doenças e as incapacidades funcionais causam impacto sobre a família, sobre o sistema de saúde e para o cotidiano dos idosos, deve-se buscar postergar sua evolução, a fim de garantir longevidade com autonomia, independência e maior qualidade de vida. ${ }^{4}$

Estudos indicam que idosos tendem a usar estratégias de autocuidado como forma de manejo de sua condição de saúde, tais como o uso de equipamentos de autoajuda (aferidores de pressão arterial, medidores de glicemia e colesterol), fracionamento das atividades ao longo da semana, buscar informações sobre saúde e bem-estar na internet, prática de exercícios físicos, entre outras. ${ }^{5,6}$

Ao longo dos anos, a população brasileira tem incorporado o uso de equipamentos de monitoramento da saúde de uso doméstico e 
para a prática de atividades físicas, conforme ocorre há vários anos com a população dos países desenvolvidos. No Brasil, esta incorporação pode ser observada em estudos realizados num projeto de inclusão digital para idosos realizado na Faculdade de Medicina de Ribeirão Preto (FMRP) da Universidade de São Paulo (USP). ${ }^{1,7}$

As pessoas têm sido cada vez mais responsabilizadas pelo autocuidado da saúde e, atualmente, o Ministério da Saúde disponibiliza monitores de glicemia, permitindo que diabéticos automonitorarem seu índice glicêmico (tarefa que no início da década de 80 era somente feita em hospitais e/ou em clínicas especializadas). ${ }^{8}$ No entanto, o automonitoramento doméstico dos diabéticos, ou seja, as constantes medidas de níveis glicêmicos por meio da utilização do medidor de glicemia, tem sido comprometido pela dificuldade no manuseio do aparelho, na aquisição de fitas, lancetas ou mesmo do próprio aparelho; tempo (para fazer os teste e anotar o resultado em planilhas ou cadernos), dificuldade na compreensão da necessidade desse monitoramento, além da dor ocasionada pelo furo na ponta do dedo para retirada de sangue e da falta de apoio familiar.

O automonitoramento também está disponível para os hipertensos, com os medidores de pressão arterial; para os cardíacos, com o frequencímetro, para uso nas atividades físicas; ou para qualquer pessoa que queira utilizá-los independentemente de possuir alguma doença crônica.

Considera-se que equipamentos tecnológicos como aferidor de pressão arterial, frequencímetro e medidor de glicemia podem auxiliar no autocuidado à saúde e podem ser aliados no controle de condições crônicas e também na qualidade de vida do usuário. Esses equipamentos têm sido francamente importados para o Brasil e têm chegado ao usuário final de forma desorientada.

No Brasil, a Agência Nacional de Vigilância em Saúde (ANVISA) e seus Núcleos de Avaliação de Tecnologias em Saúde (NATS) têm importante ação neste sentido, assim como os profissionais de saúde que irão orientar seu uso doméstico. ${ }^{10}$ Como a maioria dos aparelhos oferece respostas "acessíveis e diretas", o maior risco é o usuário tentar o autodiagnóstico e o autotratamento. "Eles podem ser excelentes aliados do monitoramento de alguns sinais, mas não devem ser usados sozinhos e sem supervisão". 11

Destaca-se a importância de desenvolver a competência para o manejo desses equipamentos e a adequada orientação a quem vai utilizá-lo, buscando esclarecer que estes não substituem a necessidade dos exames clínicos e de equipamentos mais confiáveis para a fase de diagnóstico, nem das consultas regulares para a fase de acompanhamento.

O desenvolvimento de sistemas mais avançados para o cuidado à saúde que envolve o cuidado à distância perpassa, em primeira instância, a competência do sujeito para o manejo dessas interfaces. Assim, o uso de equipamentos de cuidados à saúde, a prática de uso do alarme do aparelho celular para indicar a hora do remédio, dentre inúmeras possibilidades, implicam conhecer o âmbito das dificuldades e variáveis que interferem no uso de equipamentos.

Em face desse panorama, este estudo buscou identificar os aparelhos de monitoramento da saúde frequentemente utilizados por idosos em ambiente doméstico, bem como descrever as dificuldades apresentadas para seu manejo.

\section{METODOLOGIA}

Trata-se de estudo exploratório, analítico e transversal, realizado no período de agosto de 2011 a agosto de 2012, tendo sido aprovado pelo Comitê de Ética e Pesquisa do Hospital das Clínicas da Faculdade de Medicina de Ribeirão Preto (processos no 1104/2010 e 949/2012).

A amostra incluiu 185 idosos ativos com doenças crônicas que participavam do Projeto de Inclusão Digital de Idosos (PIDI) da Faculdade 
de Medicina de Ribeirão Preto-USP. Foram excluídos os sujeitos que relataram déficits cognitivos, que apresentaram dependência total nas atividades instrumentais de vida diária, com limitação motora de membros superiores e déficit visual não corrigido e relatado (cegueira ou baixa visão).

Foram utilizados como instrumentos de coleta de dados um questionário socioeconômico semiestruturado com perguntas sobre as características dos sujeitos e das condições gerais de saúde, desenvolvido com base nos questionários socioeconômicos do $\mathrm{IBGE}^{12}$ e do Exame Nacional de Desempenho de Estudantes (ENADE); ${ }^{13}$ a Escala de Lawton \& Brody, ${ }^{14}$ validada no Brasil por Santos \& Virtuoso Júnior, ${ }^{15}$ e o questionário sobre a percepção dos idosos em relação ao uso de equipamentos de cuidado à saúde, dificuldades e estratégias elaborado pelos autores da pesquisa.

A Escala de Lawton \& Brody ${ }^{14}$ é composta de uma metodologia de pontuação de oito domínios de funções que avaliam tarefas diárias, tais como: capacidade de utilizar o telefone, realização de compras, preparo de refeições, desempenho de tarefas domésticas, lavagem de roupa, uso de meio de transportes e responsabilidade em relação a medicação e gestão dos assuntos econômicos. A pontuação de cada questão varia de 0 a 1 ponto, de acordo com o nível de independência na realização da atividade, totalizando 8 pontos para nível máximo de independência. As mulheres apresentam pontuação variando de 0 (baixa função/dependência) a 8 (alta função/ independência); e os homens, variando de 0 (baixa função/dependência) a 5 (alta função/ independência).

Três categorias foram excluídas na pontuação masculina, pois são relacionadas a atividades domésticas geralmente realizadas por mulheres. ${ }^{16}$ Fez-se necessário criar uma pontuação mínima para cada um dos gêneros, para fins de seleção dos sujeitos. Considerou-se que estariam inclusas as mulheres com pontuação igual ou maior a 4 (para uma pontuação máxima na escala para independência que é 8) e homens com pontuação igual ou maior a 3 (para uma pontuação máxima da escala para independência que é 5). Pontuações menores foram ponderadas como de alto nível de dependência.

O questionário utilizado neste estudo para o levantamento dos dispositivos usados por idosos era composto de 12 dispositivos (telefone celular, computador, tablet, leitor de texto digital, televisão, máquina fotográfica e filmadora, forno de micro-ondas, máquina de fazer pão, aparelho de DVD), sendo que três deles eram considerados dispositivos de saúde (aferidor de pressão arterial digital, medidor de glicemia digital e organizador de medicamentos eletrônico/digital). Devido ao objetivo do estudo, as análises foram focadas nos dispositivos de saúde.

Todos os idosos participantes foram esclarecidos quanto a sua participação no estudo, conforme descrito no Termo de Consentimento Livre e Esclarecido (TCLE). Ao concordarem com os termos da pesquisa, assinaram o TCLE, de acordo com a Resolução CNS n 196/96.

\section{RESULTADOS}

\section{Caracterização dos sujeitos em relação ao status socioeconômico}

A amostra incluiu 185 idosos ativos com média de idade de 70 anos, dos quais 78,4\% eram do sexo feminino e $21,6 \%$ do sexo masculino. Quanto ao estado civil, 53\% eram casados e $73,5 \%$ viviam com o cônjuge ou familiares (filhos, netos, irmãos). Em relação à escolaridade, 31,9\% possuíam ensino superior completo e apenas dois entrevistados $(1,1 \%)$ não possuíam escolaridade. Em relação à renda mensal familiar, 18,9\% possuíam renda com valores variando entre três e quatro salários mínimos (salário mínimo de referência $R \$ 622,00)$, segundo a tabela 1 . 
Tabela 1. Caracterização socioeconômica da amostra (n=185). Ribeirão Preto-SP, 2012.

\begin{tabular}{|c|c|c|}
\hline Gênero & $\mathrm{n}$ & $\%$ \\
\hline Feminino & 145 & 78,4 \\
\hline Masculino & 40 & 21,6 \\
\hline Estado civil & $\mathrm{n}$ & $\%$ \\
\hline Casado(a) & 98 & 53 \\
\hline Viúvo(a) & 46 & 24,9 \\
\hline Separação legal & 18 & 9,7 \\
\hline Solteiro(a) & 22 & 11,9 \\
\hline Outro & 0 & 0 \\
\hline Sem resposta & 1 & 0,5 \\
\hline Com quem reside & $\mathrm{n}$ & $\%$ \\
\hline Com familiar & 136 & 73,5 \\
\hline Sozinho(a) & 45 & 24,3 \\
\hline Outra situação & 2 & 1,1 \\
\hline Sem resposta & 2 & 1,1 \\
\hline Escolaridade & $\mathrm{n}$ & $\%$ \\
\hline Sem escolaridade (analfabeto) & 2 & 1,1 \\
\hline Ensino fundamental incompleto & 37 & 20 \\
\hline Ensino fundamental completo & 18 & 9,7 \\
\hline Ensino médio incompleto & 20 & 10,8 \\
\hline Ensino médio completo & 30 & 16,2 \\
\hline Ensino superior incompleto & 15 & 8,1 \\
\hline Ensino superior completo & 59 & 31,9 \\
\hline Mestrado ou doutorado & 4 & 2,2 \\
\hline Renda familiar mensal da casa & $\mathrm{n}$ & $\%$ \\
\hline Inferior a um salário mínimo & 1 & 0,5 \\
\hline Até um salário mínimo & 19 & 10,3 \\
\hline De um a dois salários mínimos & 22 & 11,8 \\
\hline De dois a três salários mínimos & 19 & 10,3 \\
\hline De três a quatro salários mínimos & 35 & 18,9 \\
\hline De quatro a seis salários mínimos & 29 & 15,7 \\
\hline De seis a oito salários mínimos & 24 & 13 \\
\hline De oito a dez salários mínimos & 12 & 6,5 \\
\hline De 10 a 12 salários mínimos & 5 & 2,7 \\
\hline Mais de 12 salários mínimos & 15 & 8,1 \\
\hline Sem resposta & 4 & 2,2 \\
\hline
\end{tabular}




\section{Caracterização dos sujeitos em relação à capacidade funcional}

Após análise da avaliação da independência nas atividades instrumentais de vida diária (AIVDs), as mulheres apresentaram dependência em algumas, mais especificamente nos itens "realização de compras", "preparo de refeições" e "gestão de assuntos econômicos que ficavam a cargo dos cônjuges ou de outras pessoas", assim como a tarefa de "lavagem da roupa" (26,8\%). A responsabilidade em relação à medicação teve alto nível de dependência, com 23,4\% das mulheres alegando necessitar de auxílio na preparação das doses; $27,5 \%$ necessitavam de auxílio na realização de compras e na preparação e aderência à medicação (25\%) e 12,5\% necessitavam acompanhamento para uso de meios de transporte.

Tabela 2. Capacidade funcional. Ribeirão Preto-SP, 2012.

\begin{tabular}{|c|c|c|}
\hline Mulheres & $\mathrm{n}=145$ & $\%$ \\
\hline Independência na realização das AIVDs & 114 & 78,6 \\
\hline Dependência parcial & 31 & 21,4 \\
\hline Dependência total & 0 & 0 \\
\hline Homens & $\mathrm{n}=40$ & $\%$ \\
\hline Independência na realização das AIVDs & 34 & 85 \\
\hline Dependência parcial & 6 & 15 \\
\hline Dependência total & 0 & 0 \\
\hline
\end{tabular}

\section{Quanto aos problemas de saúde relatados}

Do total de participantes 76,7\% ( $\mathrm{n}=142)$ possuíam alguma patologia e foram detectados 57 tipos de doenças diferentes, levando-se em conta que $42,3 \%$ deles possuíam mais que uma $(n=142)$.

Entre as doenças relatadas, a hipertensão arterial sistêmica (HAS) (48,5\%), as doenças do sistema osteomusculares e do tecido conjuntivo $(24,6 \%)$ e diabetes $(25,3 \%)$ foram as mais referidas. Em relação às duas patologias mais frequentes, o diabetes e a HAS, 13,4\% dos sujeitos apresentavam ambas.

\section{Quanto à utilização de aparelhos de cuidado à saúde}

\section{Frequência e dificuldade de uso}

O dispositivo de saúde mais utilizado pelos idosos em ambiente domiciliar foi o aferidor de pressão arterial (38,4\% dos idosos utilizam), seguido do porta-comprimidos (15,7\%) e do medidor de glicemia (14\%). Em relação à frequência de uso, o dispositivo mais usado pelos idosos foi o porta-comprimidos $(48,3 \%$ utilizavam diariamente), seguido do medidor de glicemia (23,1\% relataram usar diariamente) e do aferidor de pressão arterial (11,5\% relataram usar diariamente). $\mathrm{O}$ aparelho que os idosos tiveram maior dificuldade de usar foi o medidor de glicose (50\% responderam ter alguma dificuldade), seguido do porta-comprimidos $(16,7 \%$ relataram ter alguma dificuldade) e do aferidor de pressão arterial (8,7\% relataram alguma dificuldade).

Sobretudo em relação ao medidor de glicemia e ao aferidor de pressão arterial, os sujeitos acreditavam não ter conhecimento para interpretar os resultados ou as informações geradas, dispositivos, conferir os dados medidos e gravar os dados das avaliações. Além disso, relataram não confiar nos resultados, preferindo recorrer ao auxílio de um profissional qualificado. Mais especificamente, quando se referiram 
ao aferidor de pressão arterial, relataram dificuldades para realizar a troca de pilhas, não saber o posicionamento correto do corpo antes de aferir a pressão para maior confiabilidade do resultado, e em que parte do corpo deve-se colocar o aparelho.

Tabela 3. Aparelhos utilizados pelos idosos, frequência de uso e dificuldade na utilização ( $\mathrm{n}=185)$. Ribeirão Preto-SP, 2012.

\begin{tabular}{lccc}
\hline & $\begin{array}{c}\text { Aferidor de pressão } \\
\text { arterial }\end{array}$ & Medidor de glicemia & Porta-comprimidos \\
\hline \multicolumn{1}{c}{ Aparelhos utilizados } & $38,4 \%$ & $14 \%$ & $15,7 \%$ \\
\hline \multicolumn{1}{c}{ Frequência de uso } & & & \\
\hline Nunca utiliza & $14 \%$ & $27 \%$ & $13,8 \%$ \\
Utiliza uma vez por mês & $31 \%$ & $23,1 \%$ & $6,9 \%$ \\
Utiliza uma vez por semana & $28 \%$ & $15,0 \%$ & $20,7 \%$ \\
Utiliza duas ou três vezes na semana & $15,5 \%$ & $4 \%$ & $3,5 \%$ \\
Todos os dias & $11,5 \%$ & $23,1 \%$ & $48,3 \%$ \\
Sem resposta & $0 \%$ & $7,4 \%$ & $6,8 \%$ \\
\hline \multicolumn{1}{c}{ Dificuldade na utilização } & & & $83,3 \%$ \\
\hline Nenhuma & $56,5 \%$ & $20 \%$ & $16,7 \%$ \\
Alguma & $8,7 \%$ & $50 \%$ & $0 \%$ \\
Muita & $8,7 \%$ & $10 \%$ & $0 \%$ \\
Não sabe usar & $4 \%$ & $0 \%$ & $0 \%$ \\
Sem resposta & $21,7 \%$ & $20 \%$ & \\
\hline
\end{tabular}

\section{DISCUSSÃO}

Foi possível verificar que aparelhos para o controle da saúde estão presentes em boa parte das residências, mostrando que os idosos estudados têm procurado nas novas tecnologias uma maneira de cuidar de sua saúde, visando a uma melhoria da qualidade de vida sem ser preciso sair de casa. O fato de se preocuparem mais com a saúde e tentar gerenciá-la sugere maior responsabilidade para o autocuidado.

Devido ao fato de os idosos de hoje pertencerem a uma geração na qual não havia tantos equipamentos eletrônicos para uso em tarefas do cotidiano, principalmente as de cuidado à saúde, e o acesso a estes ser muito difícil, esta parcela da população é uma das mais afetadas pelas mudanças tecnológicas, o que muitas vezes acarreta dificuldades. ${ }^{17}$ Os idosos apresentam dificuldades por razões cognitivas, físicas, sociais e culturais. Os equipamentos digitais se tornam cada vez menores e com mais funções, o que dificulta o acesso para este segmento, contingente com mais dificuldades sensoriais e motoras. ${ }^{18}$

Neste estudo, o aferidor de pressão foi um dos dispositivos que os sujeitos mais utilizavam e, ainda assim, alegaram não confiar no resultado do aparelho, preferindo ir a um posto médico. Com a alta incidência de portadores de diabetes, identificou-se que muitos já possuíam o medidor de glicemia, mas metade 
dos participantes do estudo relatou ter alguma dificuldade na utilização.

Não se trata apenas da dificuldade posta para o uso no âmbito das competências do idoso, mas a complexidade de equipamentos multitarefas e também a falta de ergonomia que muitos aparelhos possuem, pois raramente são desenvolvidos visando ao uso por este público. ${ }^{19}$

As dificuldades relativas ao uso de equipamentos encontradas neste estudo podem ser lidas a partir de três aspectos diferentes e correlatos. Estas se referem às dúvidas básicas como o posicionamento na hora do uso ou sobre os passos a serem seguidos para a operação do equipamento; em segundo, estão as dúvidas quanto à confiabilidade do aparelho; e em terceiro lugar, está o questionamento sobre a própria capacidade para interpretar os resultados obtidos. Embora pareça simples, o desconhecimento dos aspectos básicos de uso e como compreender os resultados pode levar a erros de leitura da própria condição ou induzi-los a tomar a decisão inadequada, como a automedicação.

Há que se considerar que a falta de conhecimento e de orientação apropriada para uso desses aparelhos pode ser um estressor capaz de provocar estados de insegurança e "nervosismos", alterar a tomada de decisão e levar a certa rejeição de dispositivos tecnológicos. ${ }^{19}$

Estudostrazemqueas maioresdificuldadesdos idosos estão nas tarefas secundárias do aparelho, como usar menus de configuração, e que este uso seria influenciado por gênero, escolaridade e contato prévio com equipamentos tecnológicos. $\mathrm{E}$ ainda, fatores como idade, nível educacional, situação financeira, gênero e, principalmente, experiência anterior com algum tipo de aparelho eletrônico, têm sido determinantes na criação de aparelhos e equipamentos que objetivam a melhoria da qualidade de vida. ${ }^{7,20,21}$

Um fator importante que deve ser levado em consideração em relação ao uso de aparelhos eletrônicos é a própria percepção do indivíduo sobre sua capacidade de utilização. No estudo realizado por Santana et al. ${ }^{7}$ em 2012, tendo como objetivo analisar a percepção sobre a capacidade para utilizar dispositivos de saúde, com amostra de 30 idosos, 56,7\% consideraram-se capazes de utilizá-los; 43,3\% referiram não saber utilizá-los; $10 \%$ relataram ter conhecimento prévio para usar dispositivos de saúde e $90 \%$ relataram não possuir conhecimento.

Dos sujeitos que já possuíam conhecimento prévio, $10 \%$ relataram ter aprendido durante a tarefa de cuidador de familiar/amigo; 16,6\% relataram ter conhecimento devido à leitura do manual de instruções; 10\% adquiriram conhecimento indo até o posto de saúde e observando os profissionais; e os demais não responderam ou não souberam responder. Em relação às situações nas quais utilizam estes aparelhos de saúde, 33,4\% relataram fazer uso para o próprio cuidado; $16,6 \%$ utilizam esses dispositivos no cuidado dispensado a outras pessoas; $10 \%$ relataram não utilizar e $40 \%$ não responderam ou não souberam responder.

Tais resultados levam à constatação de que o espaço de aprendizagem é dinâmico e diverso, e que as tarefas de cuidado de si mesmo e do outro, de observação dos procedimentos do profissional da saúde, ou lendo o manual de instruções, também são maneiras para se desenvolver habilidades para o uso destes equipamentos.

Como a população idosa cresce rapidamente, é preciso instrumentalizar os idosos para desenvolverem com autonomia os cuidados básicos à saúde. Uma dessas ações é promover o uso competente de equipamentos eletrônicos voltados para a saúde. É possível notar que há um sentimento de desconfiança em relação aos resultados emitidos pelos dispositivos tecnológicos, fazendo com que os idosos prefiram a aferição feita por um profissional. Tal observação pode estar associada ao fato de que jamais a população brasileira fora encorajada a utilizar equipamentos como estes e se sente despreparada para operá-los ou interpretar os resultados e, consequentemente proceder a uma tomada de decisão adequada. Associa-se a isto a 
falta de orientação dos profissionais da saúde aos sujeitos que vão usar esses equipamentos.

Para refletir sobre tal dificuldade, primeiro é necessário compreender que o desencorajamento para o uso advinha de uma hegemonia dos profissionais da saúde, os quais eram detentores do conhecimento sobre toda e qualquer informação relacionada ao cuidado à saúde. Destaca-se, entretanto, a preocupação que o profissional sempre teve em relação à tomada de decisão errada, como a automedicação, por exemplo.

Um segundo aspecto se relacionava ao custo desses dispositivos, que chegavam ao Brasil com preço muito elevado e eram de difícil acesso às pessoas, sendo mais encontrados em academias de ginástica (frequencímetro, pedômetro, contador de calorias), consultórios e clínicas especializadas (monitor de pressão, de glicemia, etc.). Com o aumento da produção destas tecnologias de saúde pelo mercado nacional, pela importação a custo mais acessível e com a melhora da condição financeira da população, este cenário foi sendo mudado.

Como o próprio Ministério da Saúde preconiza, é necessário investir em educação em saúde numa perspectiva dialógica, emancipadora, participativa, criativa, que contribua para a autonomia do indivíduo em relação a sua condição de sujeito, que possui direitos e é autor de sua trajetória de saúde e doença; e autonomia dos profissionais diante da possibilidade de reinventar modos de cuidado mais humanizados, compartilhados e integrais.$^{22,23}$

O empoderamento, entendido como tecnologia educacional inovadora, está diretamente relacionado a ajudar a pessoa no crescente controle sobre sua vida, abordando os campos da promoção da saúde. O desenvolvimento pessoal e social pode ser conquistado por meio da informação, da educação para a saúde e da intensificação das competências, o que permite que a população exerça maior controle sobre sua própria saúde. ${ }^{24}$
Conhecer as dificuldades do idoso no uso de dispositivos tecnológicos pode auxiliar no desenvolvimento de estratégias de instrumentalização deste público, visando não somente a seu autocuidado e desenvolvimento de responsabilidades relativas à própria saúde, mas à qualidade do cuidado que muitas vezes o idoso dispende ao outro (cônjuge, familiar ou conhecido).

O domínio dos dispositivos eletrônicos pode gerar competência para o cuidado à saúde, para a identificação de sintomas e para a necessidade de reflexão sobre a tomada de decisão, o que muitas vezes perpassa a prática da atividade física, o controle alimentar ou o uso racional de medicamentos. Ainda, pode auxiliar na tomada de decisão durante a aquisição de aparelhos mais amigáveis e adequados à população idosa e fornecer informações que possam subsidiar o desenvolvimento de produtos ergonômicos e funcionalmente mais adequados ao idoso.

As limitações deste estudo se referem à necessidade de investigar populações de idosos que vivem em áreas rurais e não somente em região urbana; incluir sujeitos com baixíssima escolaridade e analfabetos, além de incluir idosos com alteração da capacidade funcional, de modo a identificar se essas dificuldades no uso de equipamentos de monitoramento da saúde persistem ou são influenciadas por estas variáveis. Ainda sugerimos estudos longitudinais e que incluam adultos maiores, buscando identificar se as dificuldades encontradas são relativas à condição de envelhecimento do sujeito ou ao desconhecimento de como se operam tais equipamentos.

\section{CONCLUSÃO}

Em relação aos resultados encontrados neste estudo, conclui-se que o dispositivo de autocuidado a saúde mais utilizado foi o monitor de pressão arterial, muito provavelmente porque a doença que mais acomete os idosos investigados é a hipertensão arterial sistêmica (HAS). 
Apesar de os idosos se considerarem capazes de utilizar o equipamento, a maioria não tem conhecimento prévio para manuseá-lo e sente algum tipo de dificuldade, muito provavelmente por pertencer a uma geração que não conheceu essas facilidades ou que não utilizou tais aparelhos anteriormente. A falta de programas de treinamento leva os idosos a buscarem informações nos manuais de instrução do aparelho, sendo que nem sempre estas são suficientes para o autocuidado ou para o cuidado do próximo.

Os avanços da tecnologia são rápidos e crescentes, e essas mudanças são mais velozes do que a capacidade do sujeito idoso de se adaptar a elas. As dificuldades de uso não podem apenas ser relacionadas ao idoso; deve-se também observar criticamente essa necessidade de mudança, que estimula o consumismo e faz com que aparelhos eletrônicos sejam superados em tão pouco tempo. O envelhecimento da população tem impulsionado o mercado para o desenvolvimento de produtos voltados a este segmento, e isto pode beneficiá-los de alguma forma.

\section{REFERÊNCIAS}

1. Silva DO. Uso de aparelhos eletrônicos por idosos em ambientes domésticos [dissertação]. São Paulo: Escola de Engenharia de São Carlos;Faculdade de Medicina de Ribeirão Preto; Universidade de São Paulo, Instituto de Química de São Carlos; 2011.

2. Schmidt MI, Duncan BB, Silva GA, Menezes AM, Monteiro CA, Barreto SM, et al. Doenças crônicas não transmissíveis no Brasil: carga e desafios atuais. Lancet [Internet] 2011;1:61-74 [acesso em 15 Dez. 2013]. Disponível em: http://download.thelancet. com/flatcontentassets/pdfs/brazil/brazilpor4.pd

3. Laboissière P. OMS: doenças crônicas não transmissíveis são a maior causa de morte em todo o mundo [internet]. Brasília: EBC; 2011 [acesso em 15 Dez. 2013]. Disponível em: http://agenciabrasil.ebc. com.br/noticia/2011-09-14/oms-doencas-cronicasnao-transmissiveis-sao-maior-causa-de-morte-emtodo-mundo

4. Tavares DMS, Dias FA. Capacidade funcional, morbidades e qualidade de vida de idosos. Texto \& Contexto Enferm 2012;21(1):112-20.
Poucos são os estudos na literatura nacional que abordam o uso de tecnologias por idosos, especificamente o uso de tecnologias voltadas ao autocuidado com a saúde em ambiente doméstico. Sugere-se, portanto, realizar estudos com amostras mais amplas e com diferentes diagnósticos, analisando se há diferenças relacionadas a escolaridade, ocupação prévia, condição socioeconômica, entre outros aspectos. Desta forma, compreender o fator humano envolvido no binômio "equipamento eletrônico e idoso" pode ser de grande relevância no âmbito das ações voltadas à saúde da população idosa.

\section{AGRADECIMENTOS}

Este estudo foi financiado pelo Conselho Nacional de Desenvolvimento Científico e Tecnológico (CNPq), pela Coordenação de Aperfeiçoamento de Pessoal de Nível Superior (CAPES) e pela Pró-reitoria de Cultura e Extensão Universitária da Universidade de São Paulo, nas modalidades bolsa de pesquisa e auxílio.

5. Hewlett S, Cockshott Z, Byron M, Cozinha K, Papa D, Hehir M. Patients' perceptions of fatigue in rheumatoid arthritis: Overwhelming, uncontrollable, ignored. Arthritis Rheum 2005;53(5):697-702.

6. Henriques MA, Costa MA, Cabrita J. Adherence and medication management by the elderly. J Clin Nurs 2012;21(21-22):3096-105.

7. Santana MP, Bernardes MS, Raymundo TM, Santana CS. Instrumentalization program of elderly for the use of health care facilities in the domestic environment. Gerontechnology 2012;11(2):193.

8. Prefeitura Municipal de São Paulo, Secretaria Municipal da Saúde. Programa de Automonitoramento Glicêmico [Internet]. São Paulo: Prefeitura de São Paulo; 2014 [acesso em 15 Dez. 2013]. Disponível em: http://www.prefeitura.sp.gov.br/cidade/secretarias/ saude/programas/index.php?p=6070\#topo

9. Cristofidis LS, Seyffarth AS. Perfil glicêmico computadorizado: instrumento de educação nutricional em Diabetes Mellitus. Comun Ciênc Saúde 2008;19(2):145-54. 
10. Agência Nacional de Vigilância Sanitária . Boletim Informativo: Segurança do Paciente e Qualidade em Serviços de Saúde [Internet]. Brasília, DF: ANVISA; 2001 [acesso em 15 Dez 2013]. Disponível em: http://portal.anvisa.gov.br/wps/wcm/connect/ f72c20804863a1d88cc88d2bd5b3ccf0/BOLETIM+I. PDF?MOD=AJPERES

11. Vilela R. Conheça sete inovações tecnológicas para monitorar sua saúde [Internet]. 2011 [acesso em 13 Dez. 2012]. Disponível em: http://www.minhavida. com.br/saude/galerias/13164-conheca-sete-inovacoestecnologicas-para-monitorar-sua-saude.

12. Instituto Brasileiro de Geografia e Estatística. Pesquisa Mensal de Emprego de 2010 (Antiga Metodologia) [Internet]. Rio de Janeiro: IBGE; 2010 [acesso em 2013 dez 15]. Disponível em: http:// www.ibge.gov.br/home/estatistica/indicadores/ trabalhoerendimento/pme/pmemet2.shtm

13. Ministério da Educação. Sistema Nacional da Avaliação da Educação Superior,Exame Nacional do Ensino Médio (ENADE). Questionário socioeconômico. 2006 [acesso em 13 Dez. 2012]. Disponível em: http://download.inep.gov.br/ download//enade/2006/QS.pdf

14. Lawton MP, Brody EM. Assessment of older people: Self-maintaining and instrumental activities of daily living. Gerontologist 1969;9(3):179-86.

15. Santos RL, Virtuoso Júnior JS. Confiabilidade da versão brasileira da escala de atividades instrumentais da vida diária. Rev Bras Promoç Saúde 2008;21(4):290-96.
16. Graf C. The Lawton Instrumental Activities of Daily Living Scale. Am J Nurs 2008;108(4):53-62.

17. Souza D. Seminário temático: investigação qualitativa [Internet]. 2007 [acesso em 13 Jan. 2014]. Disponível em: http://www2.dce.ua.pt/leies/pacgi/ NUD'IST_24_10_2007.pdf

18. Rocha D, Deusdará B. Análise de conteúdo e análise do discurso: aproximações e afastamentos na (re) construção de uma trajetória. Alea 2005;7(2): 305-22.

19. Gamberini L, Alcaniz M, Barresi G, Fabregat M, Ibanez F, Prontu L. Cognition, technology and games for the elderly: An introduction to ELDERGAMES Project. Psychnology J 2006;4(3):285-308.

20. Goldman SN. Envelhecimento e inclusão digital. In: Freitas EV, Py L, editoras. Tratado de geriatria e gerontologia. Rio de Janeiro: Guanabara Koogan; 2007. p. 1466-72.

21. Silva DO, Raymundo TM, Santana CS. Use of electronic devices in homes by elderly. Gerontechnology 2012;11(2):192.

22. Lima-Costa MF, Veras R. Saúde Pública e envelhecimento. Cad Saúde Publica 2003;19(3):700-01.

23. Brasil. Ministério da Saúde, Secretaria de Políticas de Saúde. Brasília, DF; 2007.

24. Oliveira SHS, Monteiro MAA, Lopes MSV, Brito DMS, Vieira NFC, Barroso MGT. Estratégias de enfrentamento da pobreza e sua interface com a promoção da saúde. Rev Latinoam Enferm 2007;15:867-73. 